\title{
Espectrofotometria de lentes of tálmicas orgânicas de visão simples submetidas à radiação ultravioleta A, ultravioleta B e luz visível
}

\author{
Spectrophotometry of organic ophthalmic lenses of simple vision submitted to \\ ultra violet $A$ and $B$ radiation and visiblelight
}

\author{
Mylene Leal Matsuhara ${ }^{1}$ \\ Liliana Rocha Galvão Machado ${ }^{2}$ \\ Luciene Chaves Fernandes ${ }^{3}$
}

${ }^{1}$ Médica Oftalmologista, Coordenadora do Serviço de Visão Subnormal do Instituto de Olhos de Belo Horizonte (IOBH).

${ }^{2}$ Médica Oftalmologista, Coordenadora do Serviço de Visão Subnormal do Instituto Hilton Rocha.

${ }^{3}$ Doutora em Oftalmologia pela Faculdade de Medicina da UFMG, Coordenadora do Serviço de Visão Subnormal do Hospital São Geraldo - Universidade Federal de Minas Gerais (UFMG).

Endereço para correspondência: Mylene Leal Matsuhara - Rua Clementino Viana Dotti, 121/202.

Belo Horizonte (MG) CEP 30575-130

Email:mylenemat@terra.com.br

Recebido para publicação em 25.04.2003

Versão revisada recebida em 30.09.2003

Aprovação em 20.01.2004

Nota Editorial: Pela análise deste trabalho e por sua anuência na divulgação desta nota, agradecemos aos Drs. Paulo Ricardo de Oliveira e Edison João Geraissate Filho.

\section{RESUMO}

Objetivo: Verificar, por meio de estudo espectrofotométrico, o poder de filtração da radiação ultravioleta A (UVA) e B (UVB) e transmitância da luz visível em lentes incolores de visão simples. Métodos: Análise espectrofotométrica (espectrofotômetro modelo Humphrey Lens Analyzer LA 360) de 14 tipos de lentes oftálmicas incolores, confeccionadas em material orgânico de diferentes características. Cada tipo de lente foi representado por 20 exemplares, totalizando amostra de 280 lentes estudadas. Posteriormente procedeu-se a análise da curva de transmitância na faixa do espectro entre 290 e 700 nm. Resultados: Em relação à radiação UVB, todas as lentes estudadas apresentaram transmitância de 0 a 1\%. Tiveram melhor desempenho (0\% de transmitância): Trivex, Rugged Fashion Wear, AO Lite, Hard Resin, Sola Light 1.56, MAR 1.56, MAR CR39, Stylis Crizal, Crizal, AirWear e Orma 15. Quanto à radiação UVA a transmitância oscilou de 1 a 18\%. Tiveram melhor desempenho (transmitância de 1\%): Miolight anti-reflexo 1.56, Trivex, Miolight Excellence, Rugged Fashion Wear, Sola Light, MAR 1.56, Stylis Crizal e Air Wear. Nenhuma das lentes estudadas filtrou completamente a radiação UVA. Todas as lentes estudadas apresentaram transmitância superior a 95\% em relação ao espectro visível, havendo variação de 95 a 100\%. Conclusões: Todas as lentes estudadas apresentaram poder de filtração UVB de 99 a 100\%, UVA de 82 a 99\% e 95 a 100\% de transmitância da luz visível. A presença de tais filtros em lentes incolores amplia as possibilidades de prescrição deste recurso de proteção ocular.

Descritores: Espectrofotometria ultravioleta/métodos; Lentes; Radiação; Luz

\section{INTRODUÇÃ̃O}

Existem três tipos de radiação ultravioleta: UVA (315-380 nm), UVB (280$315 \mathrm{~nm}$ ) e UVC (100-280 nm). A principal fonte de radiação ultravioleta é o sol, porém não devemos nos esquecer das luzes artificiais (incandescentes e fluorescentes). Já a luz visível se situa no espectro de 381 a 760 nm² .

Diante da progressiva destruição da camada de ozônio, com uma perda estimada de $12 \%$ por década, aumenta a preocupação em prescrever lentes filtrantes $^{(2)}$. A relação de causa e efeito entre a radiação UV, luz visível e a ocorrência de danos oculares vêm sendo descrita em vários estudos realizados nos últimos anos ${ }^{(3-7)}$. Doenças oculares como pterígio, fotoceratites, uveítes anteriores e catarata estão associadas à exposição às radiações UVA e UVB, enquanto alterações retinianas como degeneração macular 
relacionada à idade (DMRI) parecem estar relacionadas além da radiação UVA e UVB, a uma porção do espectro da luz visível (luz azul) ${ }^{(3-8)}$.

O uso de filtros de raios ultravioleta (UV) veio incorporarse ao crescente aprimoramento dos materiais utilizados na fabricação de lentes oftálmicas. Assim, a cada dia, a indústria óptica procura lançar uma nova lente com maior capacidade de filtração.

O objetivo do presente estudo foi verificar, através do estudo espectrofotométrico, a presença e intensidade do poder de filtração dos raios UV e transmitância da luz visível em lentes incolores de visão simples atualmente disponíveis no mercado nacional com o propósito de orientar o oftalmologista na escolha da melhor lente a ser prescrita.

\section{MÉTODOS}

Foram utilizados 14 tipos de lentes oftálmicas de diversos fabricantes, confeccionadas em material orgânico de diferentes características, no que se refere ao índice de refração, índice ABBE e tratamentos (Quadro 1).

A amostra foi constituída de 20 exemplares de cada tipo de lente estudada, totalizando 280 lentes. Para manter a uniformização da amostra e reduzir as variáveis, todas as lentes eram incolores, de visão simples e sem poder dióptrico.

Cada lente foi avaliada quanto a sua transmitância às radiações ultravioleta A e B e à luz visível, em um mesmo aparelho de espectrofotômetro (modelo: Humphrey Lens Analyzer LA 360). Este aparelho possuía como características: espectro de leitura entre 290 a $700 \mathrm{~nm}$, resolução de transição de até $1 \%$, possibilidade de leitura em lentes entre 7 a 100 mm de diâmetro. A leitura espectrofotométrica foi realizada na porção central e face externa de cada lente individualmente.

Posteriormente procedeu-se a análise da curva de transmitância de cada lente na faixa de espectro entre 290 a 700 nm.

\section{RESULTADOS}

Conforme exposto na tabela 1 no que se refere à capacidade de filtração à radiação UVB, tiveram melhor desempenho ( $0 \%$ de transmitância) as lentes: Trivex, Rugged Fashion wear, AO Lite, Hard Resin, Sola Light 1.56, MAR 1.56, MAR CR39, Stylis Crizal, Crizal, AirWear e Orma 15. Em seguida, com $1 \%$ de transmitância: Miolight anti-reflexo 1.56, Ultra Thin e Miolight Excellence.

Em relação à proteção da radiação UVA, as lentes que tiveram melhor desempenho (transmitância de 1\%) foram: Miolight anti-reflexo 1.56, Trivex, Miolight Excellence, Rugged Fashion Wear, Sola Light, MAR 1.56, Stylis Crizal e Air Wear. Em segundo lugar, com transmitância entre 6\% a 8\%, as lentes: AO Lite, Hard Resin, MAR CR39, Crizal e Orma 15. Com transmitância entre $16 \%$ a 18\%, a lente Ultrax Thin. Nenhuma das lentes estudadas filtrou completamente a radiação UVA.

Em relação à luz visível, todas as lentes estudadas tiveram transmitância superior a 95\%. Houve variação entre 95\% a 100\%.

\section{DISCUSSÃO}

Geralmente, quando se fala em lentes filtrantes, é comum a associação com lentes coloridas. Estas últimas se dividem em fotocromáticas, fotossensíveis e tingidas. Possuem além da capacidade de filtração à radiação UV, o poder de bloquear parcialmente o espectro de luz visível. Tendo assim, o objetivo de aumentar o conforto ocular através da redução do ofuscamento e da fotofobia ${ }^{(8-9)}$. Na realidade, as lentes incolores também podem filtrar a radiação UV. Isto se deve ao fato de a lente orgânica encerrar em sua composição substâncias do grupo das benzofenonas, que barram a passagem dos raios UV mesmo quando incolor ${ }^{(10)}$. Com este estudo corroboramos o conhecimento de que a capacidade de filtração à radiação ultravioleta das lentes orgânicas, independe da sua coloração.

\begin{tabular}{|c|c|c|c|c|}
\hline Lente & Material & Índice refração & Características & Índice $A B B E$ \\
\hline Miolight Excellence & CR39 & 1,60 & Anti-reflexo(verde), antiabrasão hidrorepelente, filtro UV & 41 \\
\hline Ultrax Thin & CR39 & 1,49 & Antiabrasão, hidrorepelente, anti-reflexo (verde), filtro UV & 58 \\
\hline Mioligth Anti-reflexo & CR39 & 1,56 & Antiabrasão, filtro UV anti-reflexo (azul) & 40 \\
\hline Trivex & CR39 (trivex) & 1,53 & Antiabrasão, proteção UV até 400 & 43 \\
\hline Orma 15 & CR39 & 1,50 & Filtro UV (100\% UVB, 93\% UVA) & 58 \\
\hline Airwear Crizal & Policarbonato & 1,59 & Hidrofóbica, antiabrasão, anti-reflexo (verde), filtro UV & 32 \\
\hline Crizal & CR39 & 1,50 & Antiabrasão, anti-reflexo, hidrofóbica & 58 \\
\hline Stylis Crizal & Policarbonato & 1,67 & Antiabrasão, proteção UV & 31 \\
\hline Sola Light 1.56 & CR39 & 1,56 & Asférica, proteção UV (UVB 100\%, UVA 98\%) & 40 \\
\hline Rugged Fashionwear & Policarbonato & 1,58 & Filtro UV (100\% UVA e UVB) & 30 \\
\hline AO Lite & CR39 & 1,50 & Filtro UV (100\% UVB, $91 \%$ UVA) & 58 \\
\hline MAR 1.56 & CR39 & 1,56 & Antireflexo, proteção UV (100\% UVB, 91\% UVA) & 40 \\
\hline MAR & CR39 & 1,50 & Proteção UVB 100\%, UVA 91\% & 58 \\
\hline Hard Resin & CR39 & 1,49 & Anti-risco & 58 \\
\hline
\end{tabular}


O estudo utilizou 20 exemplares de cada lente com o intuito de obter uma maior confiabilidade dos dados obtidos. Mediuse a capacidade de filtração à radiação UV e transmitância à luz visível em cada exemplar, comparando os dados obtidos com aqueles informados pelos fabricantes.

Analisando os resultados verificamos que o maior potencial de bloqueio das lentes estudadas se refere à radiação UVB, transmitância variando entre 0 a $1 \%$, do que a radiação UVA com transmitância entre 1 a 18\%. Isto é desejável e coerente com os dados da literatura, que apontam a radiação UVB como a principal porção da radiação UV relacionado com danos oculares ${ }^{(3-8)}$.

Em relação à transmitância à luz visível, devido à impossibilidade de documentação gráfica da curva de transmitância de todas as lentes estudadas, e sabendo que dentro do espectro visível existe uma porção deletéria (azul entre 380- 400 nm), não podemos afirmar que uma lente que tenha transmitância de $100 \%$ seja superior a uma com transmitância de 95\%. Sobretudo, se esta última bloquear esta porção deletéria. Novos estudos devem ser realizados neste sentido, para melhor esclarecer a questão. Contudo, todas as lentes estudadas tiveram transmitância superior a 95\%, ocorrendo variação entre

\begin{tabular}{|c|c|c|c|}
\hline Lente & $\begin{array}{c}\text { Trans. } \\
\text { UVB }\end{array}$ & $\begin{array}{c}\text { Trans. } \\
\text { UVA }\end{array}$ & $\begin{array}{l}\text { Trans. } \\
\text { luz visível }\end{array}$ \\
\hline $\begin{array}{l}\text { Miolight anti- } \\
\text { reflexo } 1.56\end{array}$ & $1 \%-20$ & $1 \%-20$ & $100 \%-20$ \\
\hline Ultrax Thin & $1 \%-20$ & $\begin{array}{l}\text { a) } 17 \%-5 \\
\text { b) } 18 \%-10 \\
\text { c) } 16 \%-5\end{array}$ & $100 \%-20$ \\
\hline Trivex & $0 \%-20$ & $1 \%-20$ & $95 \%-20$ \\
\hline $\begin{array}{l}\text { Miolight } \\
\text { Excellence }\end{array}$ & $1 \%-20$ & $1 \%-20$ & $100 \%-20$ \\
\hline $\begin{array}{l}\text { Rugged } \\
\text { Fashion Wear }\end{array}$ & $0 \%-20$ & $1 \%-20$ & $\begin{array}{l}\text { a) } 94 \%-10 \\
\text { b) } 95 \%-10\end{array}$ \\
\hline AO Lite & $0 \%-20$ & $\begin{array}{l}\text { a) } 7 \%-18 \\
\text { b) } 6 \%-2\end{array}$ & $\begin{array}{l}\text { a) } 97 \%-13 \\
\text { b) } 96 \%-7\end{array}$ \\
\hline Hard Resin & $0 \%-20$ & $\begin{array}{l}\text { a) } 7 \%-10 \\
\text { b) } 8 \%-10\end{array}$ & $\begin{array}{l}\text { a) } 97 \%-13 \\
\text { b) } 96 \%-7\end{array}$ \\
\hline Sola Light 1.56 & $0 \%-20$ & $1 \%-20$ & $\begin{array}{l}\text { a) } 4 \%-12 \\
\text { b) } 93 \%-8\end{array}$ \\
\hline MAR 1.56 & $0 \%-20$ & $1 \%-20$ & $\begin{array}{l}\text { a) } 100 \%-19 \\
\text { b) } 99 \%-1\end{array}$ \\
\hline MAR CR 39 & $0 \%-20$ & $\begin{array}{l}\text { a) } 6 \%-3 \\
\text { b) } 7 \%-16 \\
\text { c) } 8 \%-1\end{array}$ & $100 \%-20$ \\
\hline Stylis Crizal & $0 \%-20$ & $1 \%-20$ & $100 \%-20$ \\
\hline Crizal & $0 \%-20$ & $\begin{array}{l}\text { a) } 6 \%-12 \\
\text { b) } 7 \%-8\end{array}$ & $100 \%-20$ \\
\hline Air Wear & $0 \%-20$ & $1 \%-20$ & $\begin{array}{l}\text { a) } 96 \%-8 \\
\text { b) } 95 \%-12\end{array}$ \\
\hline Orma 15 & $0 \%-20$ & $\begin{array}{l}\text { a) } 7 \%-11 \\
\text { b) } 8 \%-9\end{array}$ & $\begin{array}{l}\text { a) } 96 \%-9 \\
\text { b) } 97 \%-11\end{array}$ \\
\hline
\end{tabular}

95\% a 100\%. Segundo as normas DIN em ISO 14889:1997, estão aptas para condução noturna as lentes com transmitância à luz visíveis superior a 75\%. Neste aspecto, todas as lentes tiveram valores superiores.

Apesar da capacidade filtrante a radiação ultravioleta ser apenas um dos parâmetros que devem ser valorizados na prescrição das lentes oftalmológicas, no meio de tantos outros como: índice ABBE, dispersão cromática, estética, etc; sua importância hoje se destaca, pelos conhecidos efeitos maléficos da radiação UV no nosso olho.

\section{CONCLUSÃO}

As lentes incolores estudadas demonstraram capacidade de filtração à radiação UV. Obteve-se uma maior uniformidade na capacidade de filtração à radiação UVB, transmitância variando entre 0 a $1 \%$, do que a radiação UVA com transmitância entre 1 a 18\%. O que é desejável segundo dados da literatura que apontam a radiação UVB como principal porção da radiação UV relacionada com danos oculares ${ }^{(3-8)}$.

Em relação à luz visível, todas as lentes estudadas tiveram transmitância superior a 95\%, ocorrendo variação entre 95\% a $100 \%$.

Quando analisamos em conjunto as variáveis, filtração às radiações UVB e UVA além da transmitância à luz visível, as lentes que tiveram melhor desempenho foram: Trivex, Rugged Fashion Wear, Sola light 1.56, MAR 1.56, Stylis Crizal e Air Wear.

A presença de filtro à radiação UV, também nas lentes incolores, amplia a possibilidade de prescrição deste valioso recurso de proteção ocular.

\section{AGRADECIMENTOS}

As empresas Essilor, Hoya e Sola, em especial aos seus representantes pelo fornecimento das amostras estudadas e ao Centro Ótico que gentilmente permitiu a utilização do espectrofotômetro.

\section{ABSTRACT}

Purpose: To verify, by spectrophotometric study, the filtration power of A and B ultraviolet radiation and transmittance of visible light in colorless lenses of simple vision. Methods: Spectrophotometric analysis (Model Humphrey Lens Analyzer LA 360 spectrophotometer) of 14 types of colorless ophthalmic lenses, made of organic material of different characteristics. Each lens type was represented by 20 copies, totaling a sample of 280 studied lenses. Later, the analysis of the transmittance curve in the spectrum range of 290 to $700 \mathrm{~nm}$ was performed. Results: In relation to UVB radiation, all of the studied lenses presented transmittance from 0 to $1 \%$. The ones that had a better performance ( $0 \%$ transmittance) were: 
Trivex, Rugged Fashion Wear, AO Lite, Hard Resin, Sola Light 1.56, MAR 1.56, MAR CR39, Stylis Crizal, Crizal, Air Wear, Orma 15. As for UVA rays, the transmittance oscillated from 1 to $18 \%$. The ones that had a better performance (1\% transmittance) were: Miolight Anti Reflex 1.56, Trivex, Miolight Excellence, Rugged Fashion Wear, Sola Light, MAR 1.56, Stylis Crizal e Air Wear. None of studied lenses completely filtered UVA radiation. All of the studied lenses presented transmittance over $95 \%$ in relation to the visible spectrum, with a variation from 95 to $100 \%$. Conclusions: The studied colorless lenses present a real capacity of filtration of UV radiation, especially regarding the UVB spectrum, which is favorable since this is the main portion of the spectrum related to ocular damages. All of the studied lenses presented UVB filtration power from 99 to $100 \%$, UVA from 82 to $99 \%$ and 95 to $100 \%$ of transmittance of visible light. The presence of such filters in colorless lenses increases the possibilities of prescription of this resource of ocular protection.

Keywords: Spectrophotometry, ultraviolet/methods; Lenses; Radiation; Light

\section{REFERÊNCIAS}

1. Arieta CEL. Danos oculares induzidos por raios ultravioleta [artigo na Internet].Hospital Virtual Brasileiro [citado em 2003 abr 20]. Disponível em http://www.hospvirt.org.br/oftalmologia/port/artigos/ultraviol.htm

2. Alves MS. Energia radiante. In: Alves AA. Refração.Rio de Janeiro: Cultura Médica Ltda; 1989. p.3-7.

3. Bergmanson JP, Soderberg PG. The significance of ultraviolet radiation for eye diseases. A review with comments on the efficacy of UV-blocking contact lenses. Ophthalmic Physiol Opt. 1995;15(2):83-91.

4. Gies PH, Roy CR, Toomey S, McLennan A. Protection against solar ultraviolet radiation. Mutat Res. 1998;422(1):15-22.

5. Pitts DG, Bergmanson JP. The UV problem: have the rules changed? J Am Optom Assoc. 1989; 60(6):420-4.

6. Taylor HR, West S, Munoz B, Rosenthal FS, Bressler SB, Bressler NM. The long-term effects of visible light on the eye. Arch Ophthalmol. 1992;110 (1):99-104.

7. Pitts DG, Cullen AC, Hacker PD. Ocular effects of ultraviolet radiation from 295 to $365 \mathrm{~nm}$. Invest Ophthalmol Vis Sci. 1977;16(10):932-9.

8. Silva SV, Fernandes LC, Teixeira AVNC. Espectrofotometria em lentes oftálmicas filtrantes sob radiação ultravioleta A e luz visível [monografia]. Belo Horizonte: Universidade Federal de Minas Gerais; 2000.

9. Ramos LFF, Fernandes LC, Cury LA. Espectrofotometria de lentes oftálmicas filtrantes coloridas sob radiação ultravioleta A e luz visível. In: $22^{\circ}$. Congresso do Hospital São Geraldo; 2001, Belo Horizonte, 2001.

10. Duarte A. Recursos ópticos à disposição do médico oftalmologista. Arq Bras Oftalmol. 1997; 60(3):320-31.

\title{
LV JORNADA DO CENTRO BRASILEIRO DE ESTRABISMO I ENCONTRO DOS DISCÍPULOS DO PROF. HENDERSON CELESTINO DE ALMEIDA
}

\author{
16 de oufubro de 2004 \\ Hotel Caesar Business \\ BELO HORIZONTE - MC
}

INFORMAC̣ÕES: Tel.: (31) 3489-6171 - Fax: (31) 3489-6040

E-mail: gribeiro@br.inter.net 